\title{
Endoscopic vacuum therapy for cervical leakage following esophagectomy under monitored anesthesia care: a case report
}

\author{
Seyeon Park ${ }^{1}$, Bong Soo Son ${ }^{2}$, Hyeonsoo Park ${ }^{1}$, Hye-Jin Kim ${ }^{1}$, Soon Ji Park ${ }^{1}$, Hee Young Kim ${ }^{1} \wedge$ \\ ${ }^{1}$ Department of Anesthesia and Pain Medicine, Pusan National University Yangsan Hospital, Yangsan, Republic of Korea; ${ }^{2}$ Department of Thoracic \\ and Cardiovascular Surgery, Pusan National University Yangsan Hospital, Yangsan, Republic of Korea \\ Correspondence to: Hee Young Kim. Department of Anesthesia and Pain Medicine, Pusan National University Yangsan Hospital, 20, Geumo-ro, \\ Beomeo-ri, Mulgeumeup, 50612, Yangsan, Republic of Korea. Email: anekhy@gmail.com.
}

\begin{abstract}
Although esophagectomy is a standard treatment for esophageal cancer, anastomotic leak after esophagectomy is a relatively common complication and its incidence is $10-25 \%$ for cervical anastomosis. Endoscopic vacuum therapy (EVT) is a feasible primary treatment of esophageal perforations and leaks. Currently, there are no anesthesia guidelines for EVT, however, it is usually performed under general anesthesia with endotracheal intubation, especially for cervical EVT. Here, we report a successful EVT under monitored anesthesia care (MAC) without any complication, which doesn't need to intubate the patient. A 64-year-old male with upper esophageal cancer underwent an Ivor-Lewis operation with cervical anastomosis. Vacuum assisted closure (VAC) was performed for cervical leak under general anesthesia, but there was no further improvement. Although EVT was attempted under sedation with midazolam in an endoscopy room, the procedure was discontinued because of desaturation. Furthermore, the thoracic surgeon was concerned about the possibility of dyspnea and hypoxia even after the procedure. EVT was scheduled under MAC at the request of a thoracic surgeon and medical doctor, as EVT was expected to lead to patient discomfort and difficult airway. EVT was performed successfully with no respiratory depression or patient movement using target controlled infusion with $2 \%$ propofol and remifentanil. The patient was discharged on the 78th POD without any other complications. EVT for cervical leak after esophagectomy can be successfully performed with MAC, and understanding the general condition of the patient, cooperation with the patient and the surgeon, and providing continuous oxygen supply to the patient are necessary for a successful procedure under MAC.
\end{abstract}

Keywords: Anastomotic leak; anesthesia; case report; endoscopy; esophagectomy; vacuum assisted closure (VAC)

Submitted Jul 10, 2020. Accepted for publication Sep 25, 2020.

doi: 10.21037/apm-20-1394

View this article at: http://dx.doi.org/10.21037/apm-20-1394

\section{Introduction}

Esophagectomy is the primary treatment for early stage and locoregionally advanced esophageal cancer. Anastomotic leak is a relatively common complication and its incidence is known to be $10-25 \%$ for cervical anastomosis and $3-25 \%$ for intrathoracic anastomosis (1). Further, anastomosis leak is associated with high rates of morbidity and mortality.
Treatment options include close observation, surgical reintervention, endoscopic stenting, and endoscopic vacuum therapy (EVT). EVT has been reported to be a feasible primary treatment of esophageal perforations and leaks (2).

Currently, there are no anesthesia guidelines for EVT, however, it is usually performed under general anesthesia with endotracheal intubation (3). Here, we report a case of

^ ORCID: Seyeon Park, 0000-0001-7183-1811; Hyeonsoo Park, 0000-0002-3376-3691; Hye-Jin Kim, 0000-0003-1630-0422; Soon Ji Park, 0000-0002-3417-9972; Hee Young Kim, 0000-0001-7809-8739. 


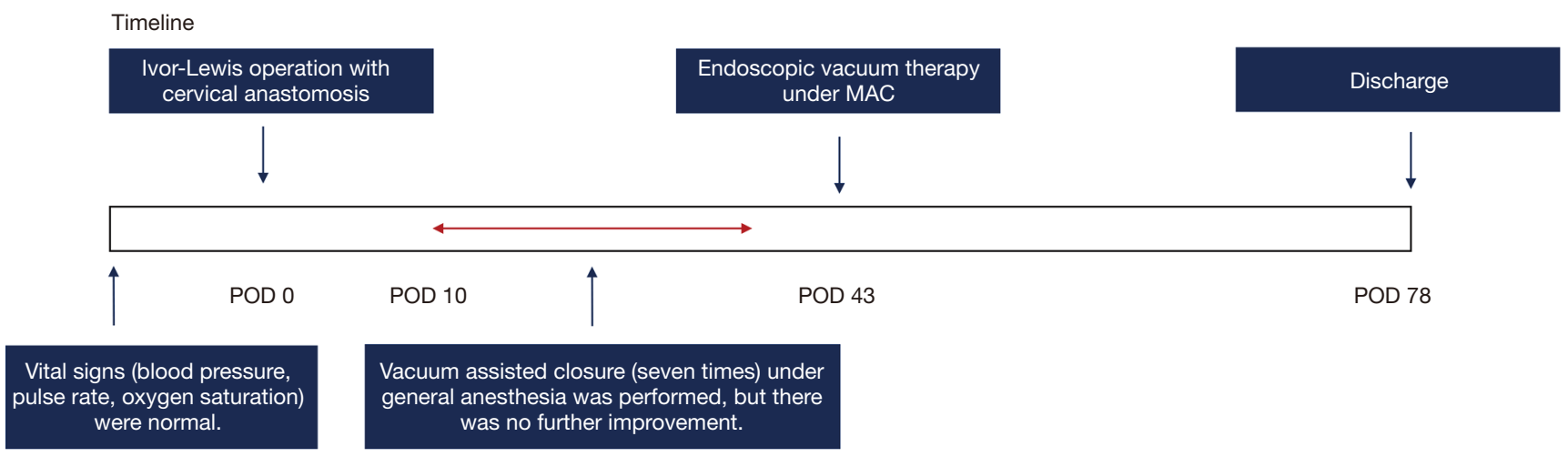

Figure 1 Timeline in this patient. Video-assisted thoracoscopic surgery for exploration and vacuum assisted closure (VAC) were performed under general anesthesia on the POD 10. After the first leakage site closure, incision and drainage and VAC-device changes were performed seven times under general anesthesia including endotracheal intubation. The patient was discharged on the 78th POD without any other complications. POD, postoperative day; MAC, monitored anesthesia care.

successful EVT under monitored anesthesia care (MAC) without any complications. We present the following article in accordance with the CARE reporting checklist (available at http://dx.doi.org/10.21037/apm-20-1394).

\section{Case presentation}

Written informed consent was obtained from the patient prior to EVT and the study conformed to the provisions of the Declaration of Helsinki (as revised in 2013). A 64-year-old male (height: $167.5 \mathrm{~cm}$, weight: $57.4 \mathrm{~kg}$ ) who was diagnosed with upper esophageal cancer and hypopharyngeal cancer underwent an Ivor-Lewis operation with cervical anastomosis and laryngomicrosurgery. Videoassisted thoracoscopic surgery for exploration and vacuum assisted closure (VAC) were performed under general anesthesia on the 10th postoperative day (POD) because of cervical anastomosis site leakage. After the first leakage site closure, incision and drainage and VAC-device changes were performed seven times under general anesthesia including endotracheal intubation (Figure 1). Nevertheless, there was no further improvement, and EVT was scheduled.

Although EVT was attempted under sedation with midazolam in an endoscopy room, the procedure was discontinued because the oxygen saturation decreased. EVT was scheduled under MAC at the request of a thoracic surgeon and medical doctor, as EVT was expected to lead to patient discomfort and difficult airway.

After entering the operating room, the hemodynamic status of the patient was stable; his blood pressure was
$109 / 75 \mathrm{mmHg}$, his heart rate was 89 beats/min, and his oxygen saturation was $96 \%$. We explained the anesthesia method to the patient and prepared devices for airway management such as an oral airway, laryngeal mask airway, endotracheal tube, direct laryngoscope, and video laryngoscope. Dexmedetomidine was administrated intravenously for about 10 minutes with $60 \mathrm{mcg}$ under $3 \mathrm{~L} / \mathrm{min}$ of oxygen supply via a nasal cannula, and was infused at a rate of $1 \mathrm{mcg} / \mathrm{kg} / \mathrm{hour}$ after the patient was appropriately sedated and his respiratory status had been checked. Remifentanil was also continuously infused ( $1 \mathrm{ng} / \mathrm{mL}$; effect-site concentration) using a targetcontrolled infusion (TCI) pump. When the gastrointestinal endoscope was inserted into the EVT site for exploration, there was a lot of movement and repeated gag reflexes from the patient. Next, $2 \mathrm{mcg} / \mathrm{mL}$ of $2 \%$ propofol and $1.5 \mathrm{ng} / \mathrm{mL}$ of remifentanil were infused using TCI, and the administration of dexmedetomidine was stopped. EVT was performed successfully, because there was no respiratory depression or patient movement (Figure $2 A, B$ ).

The oxygen saturation was maintained at $96-99 \%$, with $30 \mathrm{mmHg}$ of end-tidal carbon dioxide and a $60-63$ bispectral index. The duration of the procedure was 50 minutes, and the total duration of anesthesia was 1 hour. Intravenous ketorolac $(30 \mathrm{mg})$ was administered at the end of the operation and the patient was transferred into the post-anesthesia care unit after confirmation of his alertness and spontaneous breathing. The patient was discharged on the 78th POD without any other complications (Figure 1). 
A
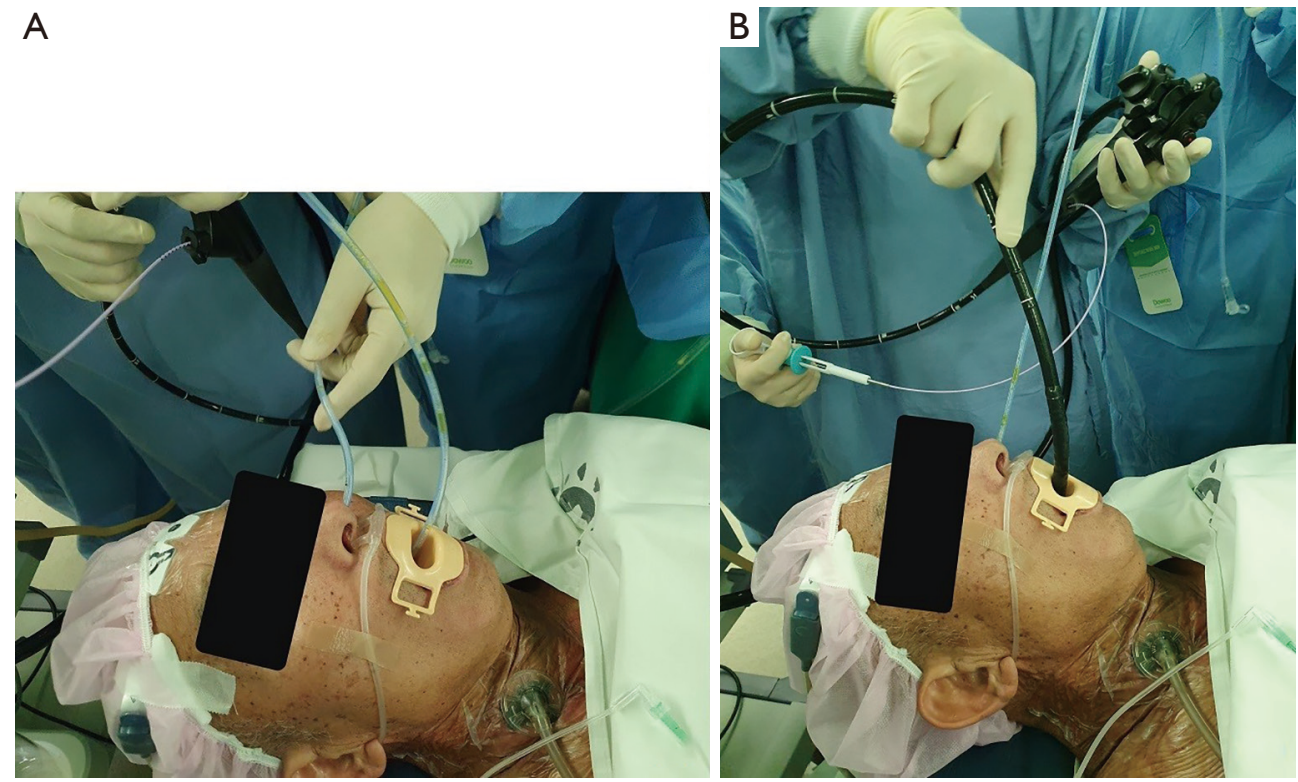

Figure 2 Endoscopic vacuum therapy (EVT). (A) A nasogastric tube is inserted via the nose and brought out through the mouth; (B) the endoscope was inserted with intracavitary placement of the tip of a size-adjusted polyurethane sponge sutured to a nasogastric tube.

\section{Discussion}

Although esophagectomy is a standard treatment for esophageal cancer, the incidence rate of anastomotic leak is reported to be up to $50 \%$ after esophagectomy (4). Anastomotic leak is sometimes asymptomatic, but may cause fulminant sepsis, contamination of the pleural space, and gastric necrosis in severe cases. In addition, anastomotic leak is the most important and feared complication, and is associated with prolonged hospital stay, stricture formation, and postoperative dysphagia (1). In addition, it accounts for approximately $40 \%$ of postoperative mortality (5).

The incidence and severity of anastomotic leaks vary depending on the conduit type, anastomotic location, and the patient's preoperative functional status. Thoracic anastomotic leaks may progress to mediastinitis and sepsis, which may require percutaneous or surgical drainage, and even a thoracotomy. However, thoracic anastomotic leaks can usually be managed with conservative treatment (4). Cervical anastomosis, on the other hand, is associated with higher rates of leaks because of the subclinical vascular insufficiency of the conduit. However, it has lower mortality rate. Cervical leaks are drained through cervical incisions, usually confined to the neck, and are thus not associated with systemic sepsis. Therefore, treatment with antibiotics or daily bedside dressing changes are sufficient in most cases. However, if the amount of the leak is significant, nasogastric decompression and nutritional support may be required, and decortication or resection with diversion should be considered when the drainage alone is not sufficient to control the leakage (1).

EVT is performed by applying negative pressure by fixing a size-adjusted polyurethane sponge at the end of a nasogastric tube, grasping the sponge with a forcep using an endoscope, and placing the sponge on the lesion. Negative pressure through EVT could promote effective drainage of the leak cavity and collapse the cavity to control the source of infection. In addition to those factors, modulation of the cytokine response and angiogenesis improves tissue healing and repair (6). Recent report has found that EVT is effective for the management of postsurgical esophageal leakage (7).

EVT can be used in cervical anastomosis leaks, however, airway irritation can cause severe coughing, shortness of breath, hypoxia, and difficulty performing the procedure. In both studies by Still et al., who found that EVT was useful esophageal perforations and leaks, the procedures were performed under general anesthesia with endotracheal intubation to ensure airway protection (3). Kuehn et al. was the first to recommend general anesthesia for the first time EVT is performed to ensure the respiratory tract is protected (2).

In this case, the patient had oxygen desaturation during 
EVT of the first cervical site under sedation. Furthermore, the thoracic surgeon was concerned about the possibility of dyspnea and hypoxia even after the procedure. Therefore, MAC was planned because general anesthesia with endotracheal intubation can mask the signs of respiratory distress associated with the surgical site. According to the American Society of Anesthesiologists, MAC is defined as a planned procedure where a qualified anesthesia provider monitors the vital signs while administering appropriate medications including sedatives or analgesics and providing services related to the patient's comfort during the procedure. Although side effects such as sympatholysis, respiratory depression, and delayed emergence can be avoided with MAC compared to general anesthesia (8), patients under MAC should be monitored and managed at the same level as regional or general anesthesia, and inhaled oxygen should be supplied during procedures. In addition, continuous and cautious monitoring of respiration, circulation, and body temperature is essential because airway obstruction, hypoxia, and even lung aspiration can occur during procedure.

In the case of a postsurgical esophageal leakage at the cervical region, especially if EVT is being performed for the first time, it is recommended that the patient be sedated with general anesthesia for the safety of the patient and the ease of the procedure by securing the airway. However, the procedure can be successfully performed with MAC when there is request from surgeon or medical doctor for the safety of the patient. For a successful procedure under MAC, understanding the general condition of the patient, cooperation with the patient and the surgeon, and providing continuous oxygen supply to the patient are necessary. Equipment for airway management should be prepared because respiratory distress could occur due to airway stimulation following EVT.

\section{Acknowledgments}

Funding: This work was supported by a 2020 research grant from Pusan National University Yangsan Hospital.

\section{Footnote}

Reporting Checklist: The authors have completed the CARE reporting checklist. Available at http://dx.doi.org/10.21037/ apm-20-1394
Peer Review File: Available at http://dx.doi.org/10.21037/ apm-20-1394

Conflicts of Interest: All authors have completed the ICMJE uniform disclosure form (available at http://dx.doi. org/10.21037/apm-20-1394). The authors have no conflicts of interest to declare.

Ethical Statement: The authors are accountable for all aspects of the work in ensuring that questions related to the accuracy or integrity of any part of the work are appropriately investigated and resolved. Written informed consent was obtained from the patient and the study conformed to the provisions of the Declaration of Helsinki (as revised in 2013).

Open Access Statement: This is an Open Access article distributed in accordance with the Creative Commons Attribution-NonCommercial-NoDerivs 4.0 International License (CC BY-NC-ND 4.0), which permits the noncommercial replication and distribution of the article with the strict proviso that no changes or edits are made and the original work is properly cited (including links to both the formal publication through the relevant DOI and the license). See: https://creativecommons.org/licenses/by-nc-nd/4.0/.

\section{References}

1. Turkyilmaz A, Eroglu A, Aydin Y, et al. The management of esophagogastric anastomotic leak after esophagectomy for esophageal carcinoma. Dis Esophagus 2009;22:119-26.

2. Kuehn F, Loske G, Schiffmann L, et al. Endoscopic vacuum therapy for various defects of the upper gastrointestinal tract. Surg Endosc 2017;31:3449-58.

3. Still S, Mencio M, Ontiveros E, et al. Primary and Rescue Endoluminal Vacuum Therapy in the Management of Esophageal Perforations and Leaks. Ann Thorac Cardiovasc Surg 2018;24:173-9.

4. Lerut T, Coosemans W, Decker G, et al. Anastomotic complications after esophagectomy. Dig Surg 2002;19:92-8.

5. Alanezi K, Urschel JD. Mortality secondary to esophageal anastomotic leak. Ann Thorac Cardiovasc Surg 2004;10:71-5.

6. Glass GE, Murphy GF, Esmaeili A, et al. Systematic review of molecular mechanism of action of negative- 
pressure wound therapy. Br J Surg 2014;101:1627-36.

7. Hwang JJ, Jeong YS, Park YS, et al. Comparison of Endoscopic Vacuum Therapy and Endoscopic Stent Implantation With Self-Expandable Metal Stent in Treating Postsurgical Gastroesophageal Leakage. Medicine
(Baltimore) 2016;95:e3416.

8. Miller RD. Choice of anesthetic technique. Basics of Anesthesia, 6th Edition Elsevier Saunders 2011; chapter 14:194-5.

Cite this article as: Park S, Son BS, Park H, Kim HJ, Park SJ, Kim HY. Endoscopic vacuum therapy for cervical leakage following esophagectomy under monitored anesthesia care: a case report. Ann Palliat Med 2021;10(4):4906-4910. doi: 10.21037/apm-20-1394 